\title{
Extraction of Region of Interest through E-Learning Videos with Matlab
}

\author{
Rahul Pratap Singh \\ Department of Computer Science \\ College Of Engineering Roorkee \\ Roorkee, India
}

\author{
Parush Agarwal \\ Department of Computer Science \\ College Of Engineering Roorkee \\ Roorkee, India
}

\begin{abstract}
With the proliferation of multimedia and the web, there now exists a high demand different types of videos. E-Learning videos have remarkable concern for research field. E-Learning videos are rapidly changing the way that universities and corporations offer education and training through the web. In recent years, the acquisition and distribution of rich media content has been largely automated, however research challenges still remain for the dynamic creation of media productions for the end user experience [3]. A video has number of frames which comprise the useful information in many ways. In E-Learning videos, our "region of interest" or ROI is board part because only board part has useful information that is present as a Text. In this paper we present an elementary Technique to extract our ROI from the frame.
\end{abstract}

\section{General Terms}

Algorithm, Design, Experimentation

\section{Keywords}

Region of Interest, Content Based Image Indexing, Image Processing, Connected Components.

\section{INTRODUCTION}

Image processing is a field which faces drastic changes and increased users day by day [1]. Web based learning has been become a most concerned area in Image Processing. E-Learning is rapidly changing the way that universities and corporations offer education and training. The growth of E-Learning has been continual and sustained. This has been fuelled by developments in Information and Communication Technologies (ICT) the nuances of which are starting to reap considerable benefits in the educational and business environment [2]. Today, many universities provide E-Learning through online Web and Video courses in Engineering, Science and humanities streams. E-Learning videos are opted mostly. In recent years, the acquisition and distribution of rich media content has been largely automated, however research challenge still remain for the dynamic creation of media productions for the end user experience[3,4]. Figure 1 shows the basic design of extracting region of interest from E-Learning videos. To implement our approach we need a dataset of E-Learning videos as raw data is the primary need to work on. Here we work on a frame of the video. The frame comprises of the utile information. There are many objects present in a frame or an RGB image. Using Matlab we can separate these components and out of these components biggest one will be the ROI which we can find using our effective approach. .

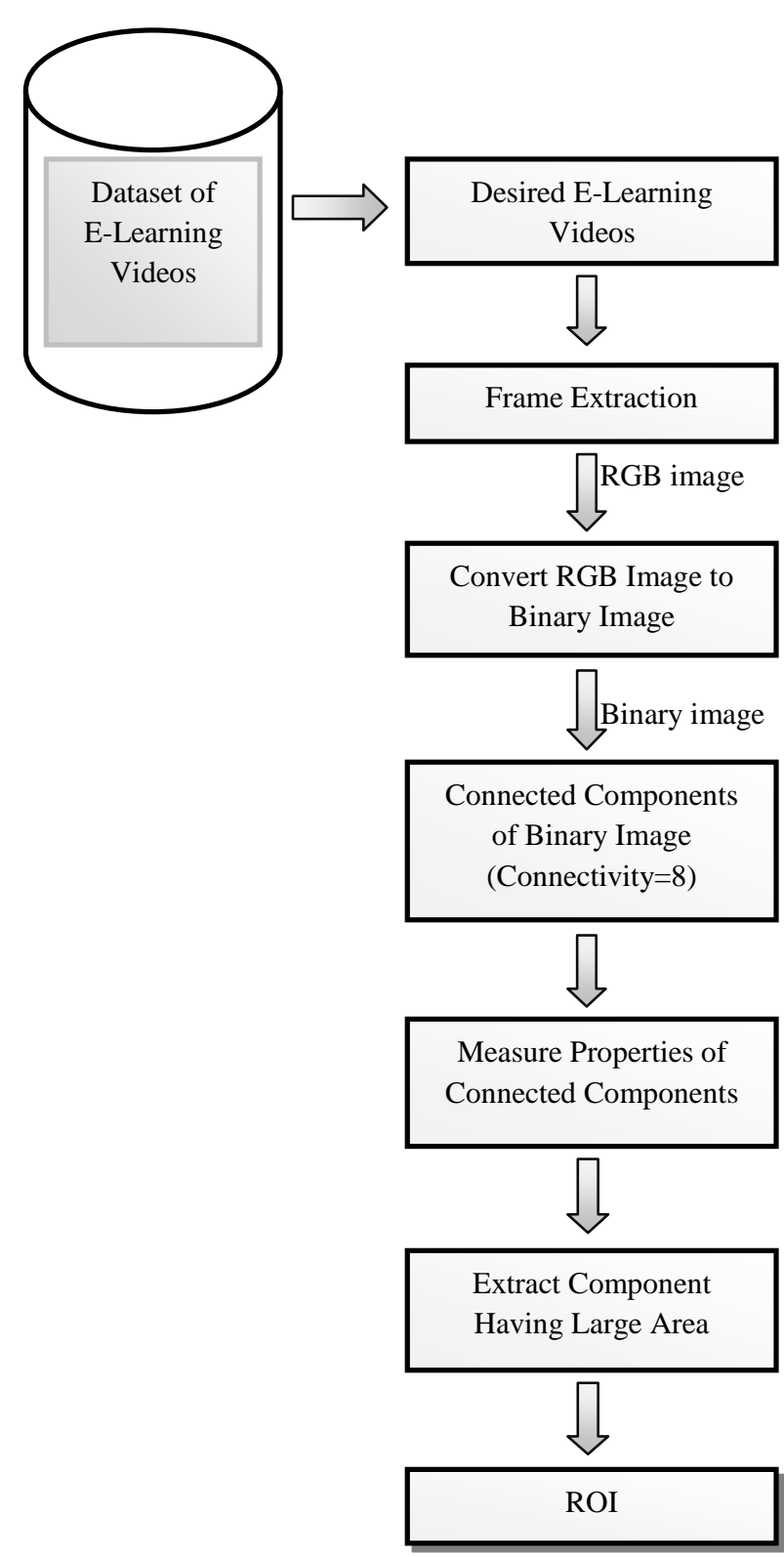

Figure 1: Working of a ROI extraction system

\section{SYSTEM DEVELOPMENT}

MATLAB is a high-level language and interactive environment for numerical computation, visualization, and programming. Using MATLAB, you can analyze data, develop algorithms, and create models and applications [6]. 
Because of these facts MATLAB has been chosen as a prototyping software.

\section{ALGORITHM}

The proposed algorithm to find ROI from an image:

Input : 2D RGB image, Threshold value

Output : ROI_file_image

variables : Binary_image, RGB_image

Subroutine main()

Binary_image $=$ im2BW $($ RGB_image, Threshold value $)$;

$\mathrm{CC}=\mathrm{BW}$ conncomp $($ Binary_image, connectivity);

reg $=$ regionProps $(\mathrm{CC}$, 'Area');

$\mathrm{ob}=$ maxobject("reg");

End Suborutine

\section{DETAILED ANALYSIS FOR PROPOSED DESIGN}

\subsection{Extraction of a Frame from the Dataset of E-Learning Videos}

A video has specific frame rate or frame frequency at which an imaging device produces unique consecutive images. Frame rates are used in synchronizing audio. In motion pictures and television, the frame rates are standardized by the Society of Motion Picture and Television Editors (SMPTE). SMPTE Time Code frame rates of 24, 25 and 30 frames per second are common, each having uses in different portions of the industry. The professional frame rate for motion pictures is 24 frames per second. So we extract a frame from the E-Learning video and use it to implement our approach. To apply the approach we have to extract a frame from an E-Learning video which works as a input RGB image. This RGB image encapsulates many subject related useful information only present in the blackboard part.

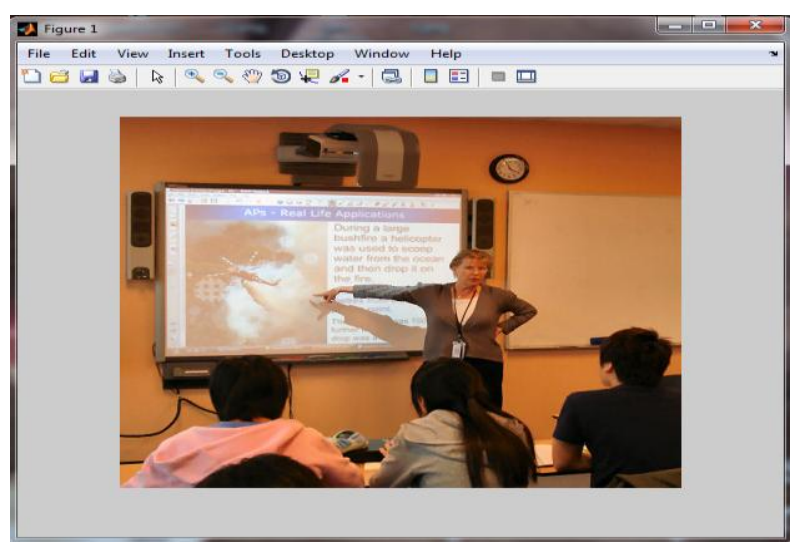

Figure 2: Input Image

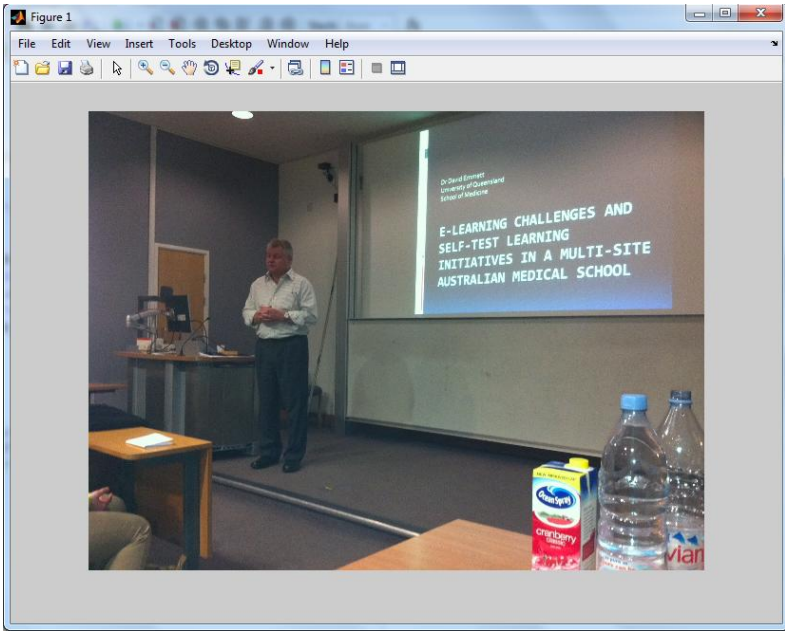

Figure 3: Input Image

\subsection{Binary Conversion of the Image}

These frames are basically images which may be in distinct format. Before uploading an image into the image database, we had to check its specifications. If the image doesn't correspond to our requirement of 256bit, we had to convert it into an image of bit depth equal to 8 . We used rgb2ind() method for achieving this. One more important prerequisite to deal with images in MATLAB is the image size. We chose the dimension $160 * 120$. We used the built-in function imresize() to automatically convert the images before uploading into the database. After uploading the images into database, RGB image is converted into binary image to attain a specific goal.

\subsection{Connected Components}

A connected component in a binary image is a set of pixels that form a connected group. For example, the binary image below has three connected components.

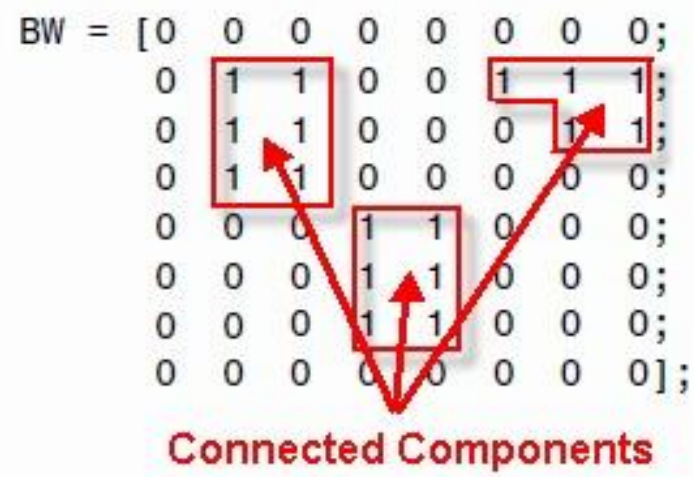

Figure 4

Connected component labeling is the process of identifying the connected components in an image and assigning each one a unique label, like this: 


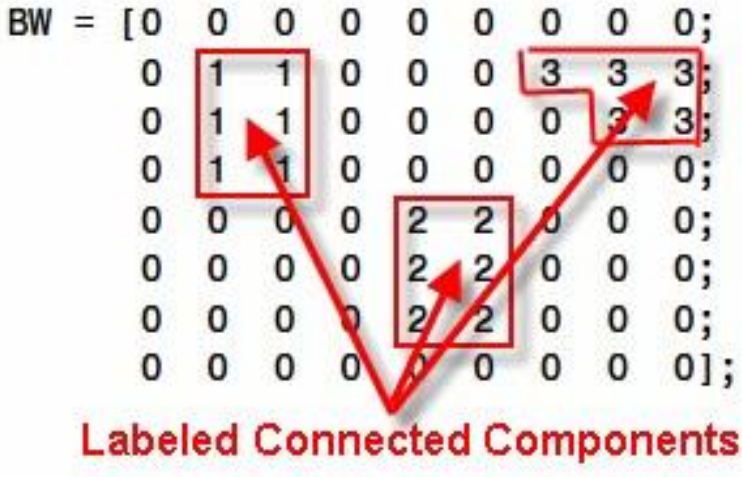

Figure 5

\subsection{ROI}

A Region of Interest, often abbreviated ROI, is a selected subset of samples within a dataset identified for a particular purpose. The concept of an ROI is commonly used in many application areas. For example, in medical imaging, the boundaries of a tumor may be defined on an image or in a volume, for the purpose of measuring its size. An ROI is often associated with categorical or quantitative information (e.g., measurements like volume or mean intensity), expressed as text or in structured form [5]. ROIs over digital images that circumscribe a desired atomic location. Image processing systems permit drawing of ROIs on images. The average parametric value is computed for all pixels within the ROI and returned to the operator. Content-based image indexing refers to the process of attaching labels to images based on their content. In E-Learning videos, the information present only in the part of blackboard portion so in this approach only that part is focused.

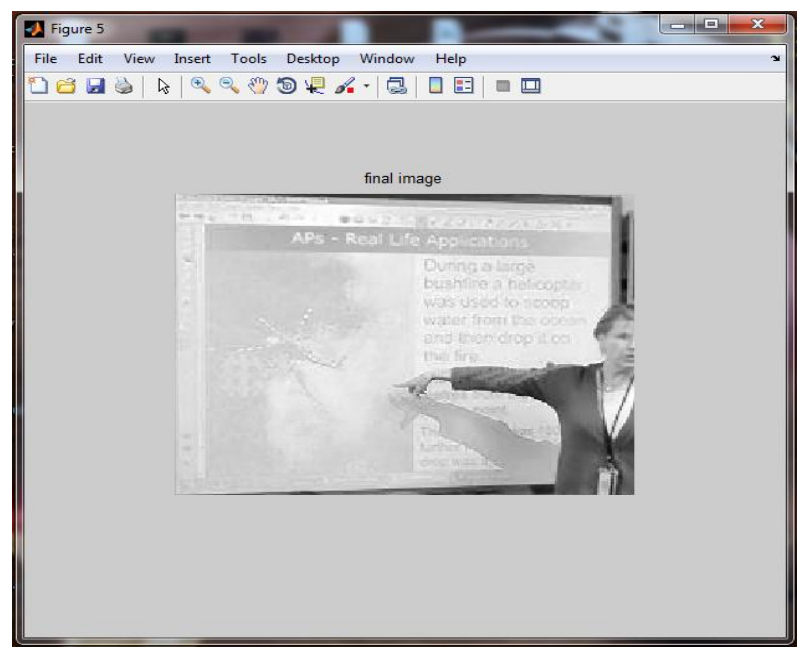

Figure 6: Desired Image (ROI)

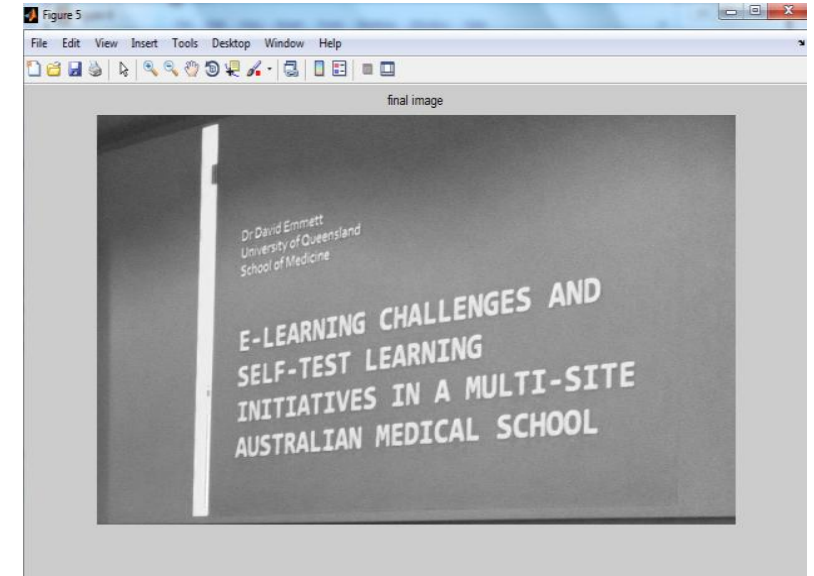

Figure 7: Desired Image (ROI)

\section{FUTURE WORK}

No functioning system can be perfect. There is always a scope of refinement. Our system is no exception. Many video lectures covers number of topic and subtopics those cannot be access retrieve without looking at the complete video. Search operation on internet related to video lectures is only based on their file name and key words assign manually to the lectures.

By extracting the text, we can add the more keywords for a particular video automatically and make a pdf file or word file such that content retrieval in the form of text related to the lectures can help in collecting the information given in lectures and can be used to study the details in pdf or documented form. We have planned to incorporate the text extraction into the current work for even more improving the retrieval efficiency.

\section{ACKNOWLEDGMENTS}

I wish to thank my guide and university for providing the consistent support for getting this project going on.

\section{REFERENCES}

[1] Shriram K V, Dr.P.L.K Priyadarsini, Subashri V "An Efficient and Generalized approach for Content Based Image Retrieval in MatLab" I.J. Image, Graphics and Signal Processing, 2012, 4, 42-48

[2] Sharifah Mazlina Syed Khuzzan, Jack Steven Goulding, Jason Underwood "Personalised learning environments : Core development issues for construction"Interactive Educational Multimedia, Number 17 (October, 2008), pp. 48-67.

[3] Mittal, A., Krishnan, P. V., \& Altman, E. (2006). Content Classification and Context-Based Retrieval System for ELearning. Educational Technology \& Society, 9 (1) , 349-358

[4] Kinshuk, \& Lin, T. (2004). Cognitive profiling towards formal adaptive technologies in web-based learning communities, International Journal of Web Based Communities, 1 (1), 103-108

[5] http://en.wikipedia.org/wiki/Region_of_Interest

[6] Matlabdefinition:http://www.mathworks.in/products/matl ab/index.html?s_cid=BB 